\title{
Peri-operative antibiotic prophylaxis in paediatric cardiac surgery
}

Keywords: Pharmacology; therapeutics; congenital cardiac malformations

Sir,

The paper by Alphonso et al. ${ }^{1}$ on peri-operative antibiotic prophylaxis in paediatric cardiac surgery is an important paper, which seeks to establish guidelines for rational use of antibiotics in this subset of patients. The guidelines are based to a large extent on the basis of previous experience in adults undergoing cardiac surgery. This is sought to be justified due to paucity of data concerning use of antibiotics after cardiac surgery in children. In the latter part of the manuscript, responses to questionnaires sent to 50 selected units worldwide have been used to arrive at conclusions.

It is here that the limitations arise. It is quite clear from Table 2 that the large majority of centres are located in the United States, and other developed countries, where the pattern of disease and presentation is entirely different from that seen in the developing world. Children in the developing world present late, are malnourished, and have more often than not sustained multiple respiratory and other infections, which render them particularly susceptible to infectious complications following open heart surgery. For example, the centre from India included in the questionnaire is beyond the reach of a vast majority of patients with congenital cardiac disease, as it is a private institution, and only patients with a reasonable socioeconomic background are able to afford such treatment. These patients are better nourished, and do not accrue the same risk of infection compared to others who belong to poor socioeconomic strata, and are exposed to multiple risk factors for infection. Antibiotics are often used for extended periods in these patients. Often their use is irrational, and promotes development of antibiotic-resistant strains of organisms. It also increases the cost of delivery of health care, which is mostly borne by the government funded health sector due to lack of health insurance.

These problems lead us to believe that local conditions in the hospitals and community have an important bearing in formulating a regime for antibiotic prophylaxis in children. The guidelines have to be further refined for those patients with severe respiratory infections, those undergoing surgery following a period of mechanical ventilatory support, and those with low body weight and in poor nutritional state. Patients with an open sternum are another subset where we need guidelines on the duration of antibiotic therapy following sternal closure.

All this will be possible only if multi-institutional trials involving a large number of patients are undertaken round the world to evolve guidelines for general practice that are based on scientific evidence on the rational use of antibiotic use in this group of patients, rather than relying on previous experience in adults. Simultaneously, in formulating these guidelines, local geographic and socioeconomic factors may be taken into consideration. Only then will it be possible to check the irrational use of antibiotics.

Sachin Talwar, Shiv Kumar Choudhary
Balram Airan
Cardiothoracic Centre
All India Institute of Medical Sciences
New Delhi, India

\section{References}

1. Alphonso N, Anagnostopoulos PV, Scarpace S, et al. Perioperative antibiotic prophylaxis in paediatric cardiac surgery. Cardiol Young 2007; 17: 12-25. 